\title{
The design of vehicle tracking based on LDC1614
}

\author{
Huang Jian \\ XiJing University, Xi'an 710123, China; \\ 565200245@qq.com
}

Keywords: Metal detection; LDC1614; vehicle tracking

Abstract: The design of an intelligent tracking car, can form thin wire along the runway running. The detection of metal adopts four channel new digital inductance sensor, each channel is connected with a self made inductance coil. In order to effectively detect the wire runway, the diameter of the self-made coil is $4 \mathrm{~cm}$. Metal detection is based on the principle of eddy current. LDC1614 can change the frequency of mutual inductance into digital output, and then send it to microprocessor STM32 by IIC serial bus to process and display. In order to realize the intelligent tracking, software programming using the mean filter algorithm, reduce interference, according to the processed data to control the car forward, turn left, turn right.

\section{Introduction}

Smart car is a hot spot this year, and has appeared many times in various electronic design competitions. For example, in 2009 the National Undergraduate Electronic Design Competition "sound guidance system" title, 2011 National University Electronic Design Competition "smart car" topic. 2016 TI cup electronic design competition, over the years "flying thinking Carle smart car" contest, there are car class topics. This competition includes science, interesting and ornamental as a whole, with the rapid development of automotive electronics, broad prospects for the background, including automatic control, pattern recognition, sensor technology, electronics, electrical, computer, mechanical and automotive and other multi-disciplinary professional creative competition.

\section{Hardware Design。}

\section{LDC1614 Hardware Design}

The schematic diagram of the LDC1614 is shown in figure 1. The 1 foot of the LDC1614 in the figure is SCL, the clock signal line in the IIC bus. The 2 foot is the SDA, the data line in the IIC bus. PE4 and STM32 are respectively connected to the PE5 pin, PE4 and PE5 as the common IO port, using IIC simulation with LDC1614 for data transmission, in order to ensure the reliability of the transmission, a $10 \mathrm{~K}$ connected to the pin on the pull-up resistor. 


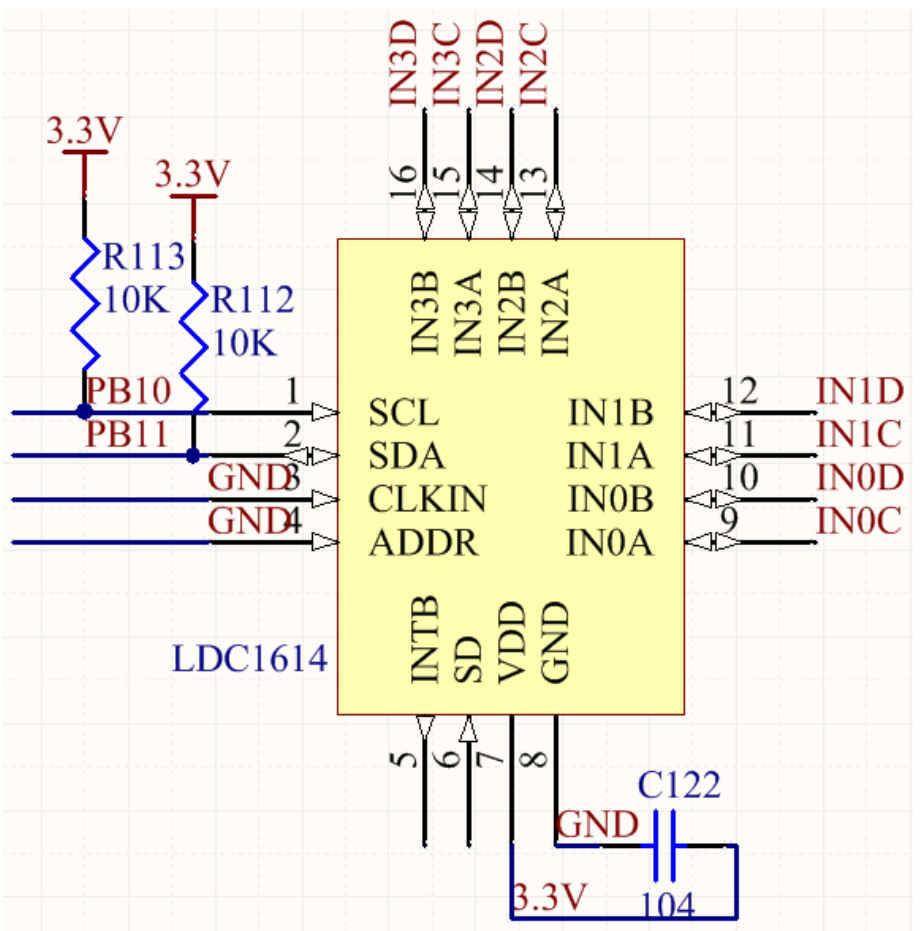

Figure 1 STM32 and LDC1614 connection diagram

\section{Motor control circuit diagram}

TB6612 is a DC motor driver produced by Toshiba formula in Japan. It is superior to L298 and can drive $2 \mathrm{DC}$ motors at the same time. The maximum current is up to $1.5 \mathrm{~A}$, and the connection diagram between TB6612 and STM32 is shown in figure 2. Due to the limited space, the article only gives some hardware circuit diagram.

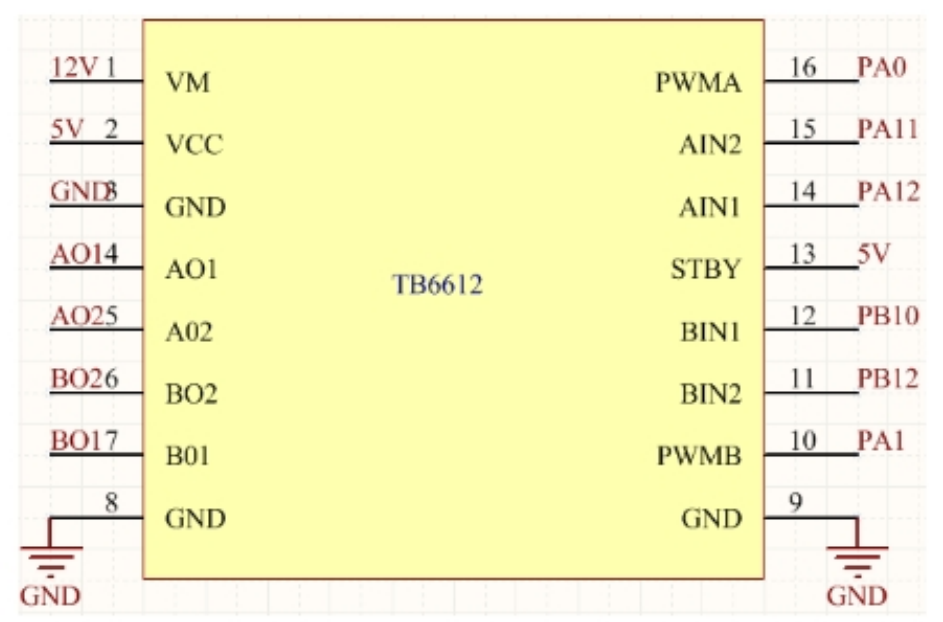

Fig. 2 Diagram of motor control circuit

\section{Software program}

In this design, under KEIL 5, programming with C language. Complete the initialization of the LDC1614 and read the four channel measurements. Some code is given below: 


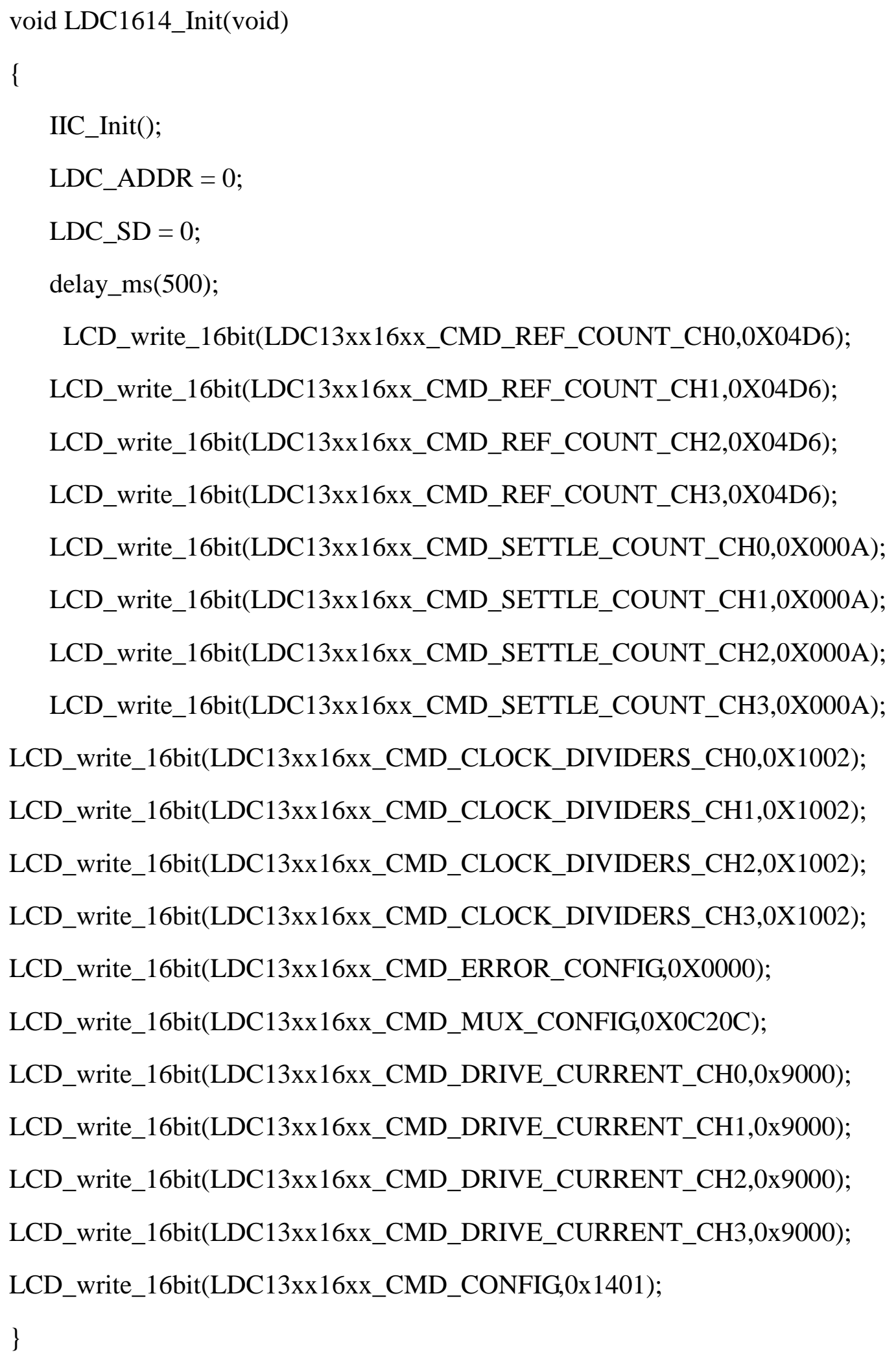

The above code implements the initialization of LDC1614 programming.

\section{Summary}

This paper designs a kind of intelligent car driving along the wire, the new LDC1614 digital inductance sensor to detect metal, configuration appropriate inductance coil and capacitance, can realize the detection of thin wire, the detection distance is close to $3 \mathrm{~cm}$. The LDC1614 is a dual channel sensor, which can realize the detection and tracking of a thin wire. 


\section{Reference}

[1] Yu Chiye, song Yue, Lei Ruiting. The research and exploration of intelligent tracking car based on STC12C5A60S2 [J]. laboratory, 2014,33 (11):46-49.

[2] Jing Wong. Research on motion control technology of smart car [D]. Wuhan: Wuhan University of Technology, 2009

[3] Yin Jie, Yang Zongshuai, Nie Hai, et al. Reflective infrared remote control car intelligent tracking system design based on J. Samson, 2013 (23): 178-184.

[4] Jin, Jia Cunliang, Wang Mei, Liu Enpeng. Intelligent tracking car design [J]. PIC microcontroller based on industrial automation, 2010, (8): 129-132.

[5] Mo Taiping, Yang Hongguang, Liu Dongmei. And the realization of [J]. automation and instrumentation, design of intelligent tracking car oriented multi route 2014, (4): 6-9.

[6] He Guifang. Design of a new intelligent metal detector [J]. instrument technology and sensor, 2016,1:13-15.

[7] teacher Zhao Bin, Guo Pengyan, Shuheng, Zou Dong. MK60N512 intelligent tracking car design based on [J]. machine and hydraulic, 2014, 42 (2): 91-96.

[8] Wang Ruru, Song Kaihong, Pan Yutian, Ming, Wu Zhenfei. Analysis of the characteristics of metal detector sensor open coil system based on [J].. Journal of HeFei University of Technology (NATURAL SCIENCE EDITION), 2015,38 (3): 354-357. 